\title{
Accuracy of five different diagnostic techniques in mild-to-moderate pelvic inflammatory disease
}

\author{
Hernando Gaitán ${ }^{1,2}$, Edith Angel ${ }^{1}$, Rodrigo Diaz $^{1}$, Arturo Parada1 ${ }^{1}$, Lilia Sanchez ${ }^{3}$ \\ and Cara Vargas ${ }^{4}$ \\ ${ }^{1}$ Obstetrics and Gynecology Department, \\ ${ }^{2}$ Clinical Epidemiology Centre and \\ ${ }^{3}$ Department of Pathology, Universidad Nacional de Colombia, Bogotá, Colombia \\ ${ }^{4}$ National Institute of Health, Colombia
}

\begin{abstract}
Objective: To evaluate the clinical diagnosis of pelvic inflammatory disease (PID) compared with the diagnosis of PID made by laparoscopy, endometrial biopsy, transvaginal ultrasound, and cervical and endometrial cultures.
\end{abstract}

Study design: A diagnostic performance test study was carried out by cross-sectional analysis in 61 women. A group presenting PID $(n=31)$ was compared with a group $(n=30)$ presenting another cause for non-specific lower abdominal pain (NSLAP). Diagnosis provided by an evaluated method was compared with a standard diagnosis (by surgical findings, histopathology, and microbiology). The pathologist was unaware of the visual findings and presumptive diagnoses given by other methods.

Results: All clinical and laboratory PID criteria showed low discrimination capacity. Adnexal tenderness showed the greatest sensitivity. Clinical diagnosis had $87 \%$ sensitivity, while laparoscopy had $81 \%$ sensitivity and $100 \%$ specificity; transvaginal ultrasound had $30 \%$ sensitivity and $67 \%$ specificity; and endometrial culture had $83 \%$ sensitivity and $26 \%$ specificity.

Conclusions: Clinical criteria represent the best diagnostic method for discriminating PID. Laparoscopy showed the best specificity and is thus useful in those cases having an atypical clinical course for discarding abdominal pain when caused by another factor. The other diagnostic methods might have limited use.

Key words: Pelvic Inflammatory Disease; Clinical Diagnosis; Laparoscopy; Endometrial Biopsy; Transvaginal Ultrasound; Endometrial Culture; Non-Specific lower Abdominal Pain (NSLAP)

Pelvic inflammatory disease (PID) is the main gynecological cause of acute lower abdominal pain $^{1}$ : prevalence has been estimated at 9-27 per 1000 fertile women ${ }^{2,3}$. In addition to immediate

Guarantors: Universidad Nacional de Colombia and International Clinical Epidemiology Network.

This study was supported by grants from: Colombian Institute for the Development of Science and Technology: COLCIENCIAS (Grant No: 075-97); Universidad Nacional de Colombia, DIB (Grant No: 809136); Bogotá Health Services: Secretaria Distrital de Salud de Bogotá; National Institute of Health Colombia; and the International Clinical Epidemiology Network: INCLEN (Grant: 1004-96-1235).

Correspondence to: H. G. Gaitán, MD, MSc, Clinical Epidemiology Centre, Universidad Nacional de Colombia, Instituto Materno Infantil, Carrera 10 \# 1-66 sur., Bogotá, Colombia. Email: hggaitan@cable.net.co, inclen@tutopia.com 
complications like peritonitis, sepsis, and death, PID may lead to consequences such as infertility, ectopic pregnancy, and chronic pelvic pain, especially in AIDS patients ${ }^{4,5}$. Such complications and consequences are related to disease severity and time of diagnosis; early diagnosis is thus essential for diminishing the disease's impact ${ }^{6}$.

PID is part of the non-specific lower abdominal pain syndrome (NSLAP) in fertile women, its symptoms representing an especially difficult challenge given the anatomical relationship between the female upper genital tract, the menstrual cycle, and pregnancy.

Clinical diagnosis is the doctor's basic tool in the emergency room for those patients presenting acute abdominal pain having no clear cause. Laparoscopy has been considered as being the gold standard for PID diagnosis ${ }^{7,8}$; but its sensitivity varies depending on the stage of the illness - being less sensitive in the mild form where diagnostic criteria are less objective ${ }^{9}$. It is not helpful for all patients (being an expensive technique), nor is it exempt from risk (being an invasive technique).

PID diagnosis is based on certain clinical criteria proposed in 1983 and modified in $1991^{10}$. These criteria have been partially evaluated. Existing studies present problems regarding the suitable selection of control population as ethical concerns emerge when an invasive gold standard such as laparoscopy is performed on a population having low probability of disease or who are healthy women. This situation has also affected evaluation of laparoscopy, ultrasound, and endometrial biopsy ${ }^{11-13}$. These techniques have not been adequately evaluated with such a goal in mind in our setting.

This study's purpose then was to evaluate the accuracy of clinical and laboratory criteria and evaluate laparoscopy, endometrial biopsy, endometrial culture, and transvaginal ultrasound performance in patients having mild-to-moderate PID, belonging to a population presenting NSLAP.

\section{SUBJECTS AND METHODS}

A diagnostic-performance test study ${ }^{14}$ was carried out by cross-sectional analysis of the patient population participating in the study to evaluate the effectiveness of laparoscopy in patients with NSLAP. Briefly, a randomized clinical trial was designed to compare the effectiveness of laparoscopy and conventional diagnosis, based on close clinical observation and paraclinical tests, in patients with NSLAP ${ }^{1}$. Patients with NSLAP were allocated to the early laparoscopy group or conventional diagnosis group using a computergenerated, random table.

The conventional diagnosis method was defined as that based on permanent clinical assessment and laboratory tests. This may have included surgical intervention such as precision laparotomy carried out by the Instituto Maternal Infantil (IMI) emergency team. PID was diagnosed by the presence of at least two of Hager's main criteria ${ }^{15}$. Appendicitis was diagnosed upon visualizing the appendix with signs of swelling or necrosis. Ectopic pregnancy was diagnosed by means of ultrasonography and serial human chorionic gonadotropin (hCG) determination or laparotomy. Ovarian cyst diagnosis was confirmed by the presence of a cystic mass in the ovary during laparotomy. Diagnosis of a healthy pelvis was reached when no alterations were found in pelvic organs during laparotomy.

The laparoscopic diagnostic method was defined as that direct visualization of the abdominal pelvic cavity, by lens, through the abdominal wall carried out by the IMI laparoscopy team, which is qualified to carry out third-level laparoscopy and has experience in lower abdominal pain diagnosis ${ }^{3}$. Laparoscopic diagnosis of PID was done according to Hager and co-workers' criteria $^{15}$. Diagnosis of unbroken ectopic pregnancy was reached by the presence of a bluish mass in the tube, whether or not associated with hemorrhagic material in the cul-de-sac ${ }^{16}$. Appendicitis and ovarian cyst diagnoses were based on visualization of those changes described in the previous paragraph. A healthy pelvis was diagnosed when no alterations were found.

Histopathologic and microbiologic tests were performed for all patients; pathologists with wide experience in gynecological disorders read the resulting histopathology. However, in none of the cases did any of the pathologists know results from laparoscopy, laparotomy, or possible etiological diagnoses of pain. 
Cases were considered to be PID when patients fulfilled at least two of the following criteria:

(a) Sight of purulent material in the upper genital tract and/or ovaries, or the presence of pus on the peritoneal surface at the bottom of the posterior sack and uterus ${ }^{7,15,17}$.

(b) The presence of chronic endometritis, according to Kiviat's criteria ${ }^{18}$.

(c) Cultures positive for Neisseria gonorrhoeae or Chlamydia trachomatis in the cervix or endometrium, or by the presence of aerobic or anaerobic bacteria, N. gonorrhoeae, mycoplasma or ureaplasma in the endometrium $^{13,19}$. (d) Presentation of acute or chronic salpingitis in pathology.

A case was considered to be non-PID when a diagnosis of appendicitis, ectopic pregnancy, complicated ovarian cyst, or endometriosis was achieved based on visual findings and pathological criteria.

Forty-nine patients then underwent visual examination (Figure 1). Nineteen of the PIDgroup and 30 of the non-PID-group patients underwent laparoscopy or laparotomy, respectively. Exclusion criteria were signs of generalized peritonitis, prior intestinal surgery, shock, chronic pelvic pain, viable intrauterine pregnancy, weight

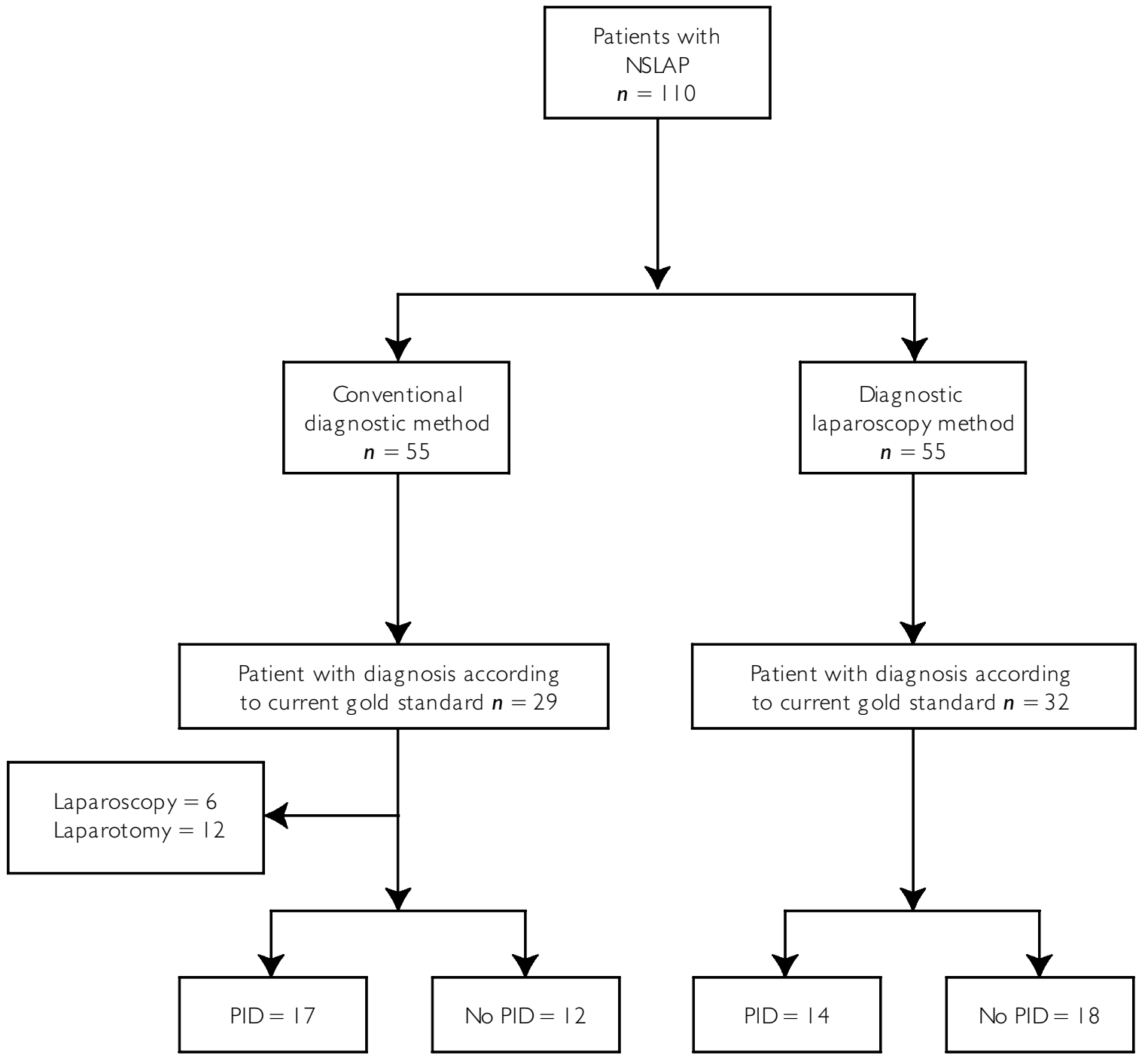

Figure I Flow chart of patients with non-specific lower abdominal pain (NSLAP). PID, pelvic inflammatory disease 
over $100 \mathrm{~kg}$ and current/previous psychiatric problems.

It was considered that patients presented a healthy pelvis when visual examination of the pelvis did not reveal any abnormal finding, the endometrial biopsy was negative for PID, and cultures were negative, with pain disappearing following the procedure.

The IMI emergency team made the diagnosis of the underlying pathology. Diagnoses in the first 6 hours following admission and 48 hours later were taken into account as clinical diagnoses.

The proposal and written consent procedures were approved by the Universidad Nacional's Ethics Committee and the Instituto Materno Infantil's Institutional Review Board.

Before the study began, the sample size was calculated by taking into account that the difference between sensitivity percentages can be used for estimating sample size in diagnostic accuracy studies $^{20}$. The EPI-INFO 6.0 statistical software was thus used to determine sample size. If it is assumed that the gold standard has $99 \%$ sensitivity and the clinical method to be compared has $70 \%$ sensitivity, then 30 patients per group are required, having 95\% significance level; the probability of a type II error was chosen to be $20 \%$ with the $\chi^{2}$ test (1:1 ratio between subjects and controls).

\section{Sample processing and transport}

Endocervical and endometrial samples were taken from all patients for detection of $N$. gonorrhoeae, C. trachomatis, and mycoplasma. Endometrial samples were also taken by Pipelle (Unimar) for histopathology and culturing of these and other aerobic and anaerobic pathogens. The exocervix was washed with saline solution prior to sample taking. A sample from the peritoneum was taken when PID was suspected during laparoscopy or laparotomy.

N. gonorrhoeae samples were also obtained from the rectum and the bottom of the cul-de-sac, when indicated. Gram stain was done and samples were prepared for transport in Thayer Martin medium in $5 \% \mathrm{CO}_{2}$ atmosphere, using the candle method. Identification and confirmatory tests were also carried out, as well as tests for $\beta$-lactamase on all isolates. Samples for C. trachomatis detection were transported in RPMI medium with bovine fetal serum and antibiotics, in refrigeration. Culturing was done in McCoy cells and colored with lugol. The samples for mycoplasma detection were transported in PPLO medium with horse serum and antibiotics, in refrigeration. Processing in PPLO agar culture with horse serum was then done. The N. gonorrhoeae, C. trachomatis, and mycoplasma samples were processed in the National Institute of Health's microbiology laboratory. The IMI microbiology laboratory processed endometrium samples for aerobic and anaerobic pathogens directly from the Pipelle in blood agar and anaerobic blood agar, respectively. A VDRL test was performed on all patients; positive cases were confirmed by using the TPHA test.

\section{Histopathology of the endometrium}

Ten percent buffered formol was used to fix samples in paraffin for staining with hematoxilyn and eosin. At least five sections were evaluated, quantifying the number of plasm cells per x120 field and glandular and stromal neutrophils per x400 field.

Transvaginal sonographic findings suggestive of PID included: liquid at the bottom of the Douglas sack; thickened fluid-filled fallopian tubes; multicystic ovaries; or anexial mass ${ }^{21}$. Ultrasound was done by the sonography team.

\section{Statistical analysis}

The groups' base socio-demographic, clinical, and laboratory variables are described below. Continuous variables were compared using Student's $t$-test or Mann-Whitney $U$ test, according to normality. Categorical variable association was evaluated by $\chi^{2}$ test. Sensitivity and specificity were evaluated for each of the clinical and laboratory criteria as well as for those methods evaluated for PID diagnosis, using STATA 6.0 software.

A PID forecast model was constructed from a multiple logistic regression model using the Stepwise method, having a 0.15 entry probability and 0.2 exit probability ${ }^{22}$. Those variables having the best discriminatory capacity in univariable analysis were then selected. The best clinical and 
Table I Base characteristics for 6 I patients having non-specific acute lower abdominal pain

\begin{tabular}{|c|c|c|c|}
\hline Characteristic & $\begin{array}{c}P I D \\
\mathrm{n}=3 I\end{array}$ & $\begin{array}{c}\text { Other pathology } \\
n=30\end{array}$ & $p$ \\
\hline Age & $27.6 \pm 7.1$ & $30.3 \pm 5.7$ & $0.11 *$ \\
\hline Gestations, mean (range) & $2(0-6)$ & $2(0-6)$ & 0.71 \\
\hline Gastrointestinal pathological antecedents & $3(9.7 \%)$ & $2(6.7 \%)$ & 0.66 \\
\hline PID antecedents & I (3.2\%) & $4(13.3 \%)$ & 0.15 \\
\hline Sexually transmitted disease antecedents & $4(12.9 \%)$ & I (3.3\%) & 0.17 \\
\hline Current use of IUD & $9(29 \%)$ & $4(13.3 \%)$ & 0.13 \\
\hline Previous use of IUD & $17(54.9 \%)$ & $12(40 \%)$ & 0.24 \\
\hline Aged less than 20 & $7(22.6 \%)$ & I (3.3\%) & 0.03 \\
\hline Current use of hormonal contraceptive & $7(22.6 \%)$ & $5(16.7 \%)$ & 0.56 \\
\hline Leukocytes & $9911 \pm 4911$ & $9597 \pm 4241$ & $0.77 *$ \\
\hline Temperature on admission & $36.6 \pm 0.6$ & $36.5 \pm 0.7$ & $0.53 *$ \\
\hline Hemoglobin & $12.7 \pm 1.7$ & $12.8 \pm 2.1$ & $0.82 *$ \\
\hline
\end{tabular}

*Student $t$-tests - other $p$-values are from Chi square tests; PID, pelvic inflammatory disease; IUD, intrauterine device

laboratory indicators were taken into account. The model's predictive capacity was evaluated through specificity, sensitivity, positive and negative predictive values, and the ROC curve.

\section{RESULTS}

A diagnosis was achieved according to the previously described criteria in 61 (55\%) of the 110 patients who presented with NSLAP between January 1998 and February 2000. The base characteristics of the group studied showed an average age of 28.5 years, $12 \%$ of such patients being less than 20 years old. Twenty percent were using an interuterine device (IUD) and $8 \%$ had a history of PID. Patients' base characteristics showed that patients having PID were younger than the control group. This difference takes on greater clinical importance when categorizing age in women older or younger than 20 . The current and past use of an IUD and the presence of sexually transmitted disease (STD) were more frequent in the PID group. None of the other characteristics showed relevant differences between the two groups (Table 1).

Patients' diagnoses can be seen in Table 2. It can be observed that there is a very low frequency of appendicitis for the type of population being studied. The prevalence of PID was 51\%.
Table 2 Exact diagnosis in patients having non-specific acute lower abdominal pain

\begin{tabular}{lc}
\hline Diagnosis & $\begin{array}{c}\text { Number of cases having } \\
\text { had exact diagnosis }\end{array}$ \\
\hline Appendicitis & 2 \\
Pelvic inflammatory disease & 31 \\
Ectopic pregnancy & 10 \\
Complex ovarian cyst & 10 \\
Endometriosis & 2 \\
Healthy pelvis & 5 \\
Urinary infection & 1 \\
Total & 61 \\
\hline
\end{tabular}

Isolated evaluation of operative characteristics for clinical criteria showed low sensitivity in all except anexial tenderness. Purulent endocervical secretion, neutrophyl count greater than $80 \%$, and abdominal rebound pain showed the best specificity. Only one patient presented with a temperature greater than $38^{\circ} \mathrm{C}$ (Table 3).

Regarding operative characteristics for diagnostic methods for PID, we found that clinical examination on admission showed the greatest sensitivity, but is not very specific; accuracy is just 69\%. This method's specificity improved with time, eventually reaching 100\%. However, clear diagnosis was only achieved by this method in 13 of 16 patients (81\%) within 48 hours of admission, with the method being used in hospitals as 
previously defined. Laparoscopy also has an optimum specificity; however, it does present some false negatives, having a $91 \%$ global accuracy.

Endometrial biopsy showed acceptable operative characteristics and 75\% accuracy. However, transvaginal pelvic ultrasound sensitivity and endometrial culture specificity were significatively

Table 3 Diagnostic operative characteristics of clinical and laboratory criteria for pelvic inflammatory disease in 61 patients having non-specific lower abdominal pain

\begin{tabular}{lcc}
\hline Criteria & $\begin{array}{c}\text { Sensitivity } \\
\text { (\%) }\end{array}$ & $\begin{array}{c}\text { Specificity } \\
\text { (\%) }\end{array}$ \\
\hline Adnexal tenderness & 93 & 6 \\
Cervical motion tenderness & 63 & 40 \\
Purulent endocervical secretion & 23 & 93 \\
Vaginal discharge & 40 & 73 \\
Leukocyte count greater than & 35 & 70 \\
$\quad$ I0 500/mm & & \\
Neutrophyl count greater than $80 \%$ & 26 & 80 \\
Abdominal rebounding pain & 25 & 90 \\
\hline
\end{tabular}

lower, having 48 and 60\% accuracy, respectively (Table 4).

There was significant isolation of anaerobic bacteria (odds ratio, OR: 4.87; 95\% confidence interval, CI: 0.83-50.2) and mycoplasma (OR 5.7; 95\% CI: 1.01-58.2) in endometrium in PID patients (Table 5). Three patients with mycolplasma in the endometrium also presented with syphilis. Another case, diagnosed with syphilis, presented an ectopic pregnancy as a complication of an underliying PID. Sensitivity was $32 \%$ and specificity rose to $90 \%$ when just isolation of anaerobic bacteria or $N$. gonorrhoeae in the endometrium was considered to be a sign of PID.

It was found that the best prediction model for PID in patients presenting with NSLAP was that involving the following variables: younger than 20 years old; current use of IUD; purulent endocervical secretion; and negative pregnancy test. The area below the ROC curve was 0.83 . This model has $69 \%$ sensitivity, $88 \%$ specificity, $86 \%$ positive predictive value, $74 \%$ negative predictive value, $79 \%$ accuracy, and $50 \%$ prevalency

Table 4 Sensitivity analysis of operative characteristics for five different techniques in the diagnosis of pelvic inflammatory disease in patients having non-specific lower abdominal pain at the Instituto Materno Infantil, Bogotá between 1998 and 2000

\begin{tabular}{lcc}
\hline & \multicolumn{2}{c}{ Diagnostic accuracy } \\
\cline { 2 - 3 } Method & Sensitivity & Specificity \\
\hline Clinical diagnosis on admission & $87 \%(27 / 3 \mathrm{I})$ & $50 \%(15 / 30)$ \\
$95 \% \mathrm{Cl}$ & $(70-96)$ & $(30-68)$ \\
Conventional diagnosis (48 hours) & $83 \%(15 / 18)$ & $100 \%(11 / 1 \mathrm{I})$ \\
$95 \% \mathrm{Cl}$ & $(58-96)$ & $(71-100)$ \\
& & $100 \%(20 / 20)$ \\
Laparoscopy & $81 \%(13 / 16)$ & $(71-100)$ \\
$95 \% \mathrm{Cl}$ & $(54-96)$ & $84 \%(26 / 30)$ \\
Endometrial biopsy & $74 \%(20 / 3 \mathrm{I})$ & $(65-94)$ \\
$95 \% \mathrm{Cl}$ & $(55-88)$ & $67 \%(20 / 30)$ \\
Transvaginal pelvic ultrasound & $30 \%(9 / 30) *$ & $(47-82)$ \\
$95 \% \mathrm{Cl}$ & $(14-49)$ & $26 \%(9 / 30) *$ \\
Endometrial culture & $83 \%(28 / 3 \mathrm{I})$ & $(12-45)$ \\
$95 \% \mathrm{Cl}$ & $(66-94)$ & \\
\hline
\end{tabular}

${ }^{*} p<0.05 ; \mathrm{Cl}$, confidence interval 
Table 5 Number of patients with surgical visualization of pelvis and endometrial results according to PID or not PID group

\begin{tabular}{|c|c|c|c|c|c|c|c|c|c|}
\hline & & & & $\begin{array}{l}\text { Endocervical } \\
\text { culture }\end{array}$ & $\begin{array}{c}\text { Endocervical } \\
\text { culture }\end{array}$ & $\begin{array}{l}\text { Endometrial } \\
\text { culture }\end{array}$ & $\begin{array}{c}\text { Endometrial } \\
\text { culture }\end{array}$ & $\begin{array}{l}\text { Endometrial } \\
\text { culture }\end{array}$ & $\begin{array}{c}\text { Endometrial } \\
\text { culture }\end{array}$ \\
\hline Group & Laparoscopy & Laparotomy & $\begin{array}{c}\text { Chronic } \\
\text { endometritis }\end{array}$ & $\begin{array}{c}\mathrm{N} . \\
\text { gonorrhoeae }\end{array}$ & Mycoplasma & $\begin{array}{c}\mathrm{N} . \\
\text { gonorrhoeae }\end{array}$ & $\begin{array}{l}\text { Aerobic } \\
\text { bacteria }\end{array}$ & $\begin{array}{c}\text { Anaerobic } \\
\text { bacteria }\end{array}$ & Mycoplasma \\
\hline $\operatorname{PID} n=31$ & 16 & 4 & 20 & 4 & II & 3 & 17 & 8 & 9 \\
\hline $\begin{array}{l}\text { No PID } \\
\quad n=30\end{array}$ & 20 & 7 & 4 & 0 & 3 & 0 & 20 & 3 & 2 \\
\hline
\end{tabular}

PID, pelvic inflammatory disease

Table 6 PID prediction model for patients having non-specific acute lower abdominal pain in patients attending the Instituto Materno Infantil, Bogotá 1998-1999

\begin{tabular}{lcc}
\hline Clinical criteria & $\begin{array}{c}\text { Adjusted } \\
\text { odds ratio }\end{array}$ & $\begin{array}{c}95 \% \text { confidence } \\
\text { interval }\end{array}$ \\
\hline Less than 20 years of age & 6.32 & $0.63-63.2$ \\
IUD currently being used & 6.4 & $1.12-36.4$ \\
Purulent cervical secretion & 6.7 & $0.75-78.6$ \\
Pregnancy test $(-)$ & 28.1 & $1.59-497.3$ \\
\hline
\end{tabular}

PID, pelvic inflammatory disease; IUD, intrauterine device

(Table 6). Operative characteristics can be seen in Figure 2.

\section{DISCUSSION}

The search for safe interventions taking available resources into account when establishing diagnosis is a priority for safe patient care in institutions. This statement becomes more forceful when considering the high costs of health attention currently sustained by employing cutting-edge technology, and the limited health-service resources available for attending patients.

The case of NSLAP represents a good scenario for evaluating the different diagnostic techniques due to the high degree of uncertainty faced by clinical diagnosis. This situation has led to the diagnostic methods studied here being used without suitable prior evaluation of technology, either in terms of effectiveness (understood as being the appropriate use of an intervention in a given situation $^{1,23}$ ) or in terms of diagnostic accuracy.

Evaluation of diagnostic criteria has been limited by the difficulty of finding a control group allowing operative characteristics to be contrasted with clinical signs. Most studies have been carried out on a population of patients submitted to laparoscopy with clinical suspicion of PID, comparing patients in whom diagnosis had been confirmed (true positive result) with those in whom a different pathology was found (false positive result). They have also described the behavior of diagnostic criteria in patients having a confirmed PID diagnosis. The number of successes (true positive result) and mistakes (false negative result) can thus be estimated for a clinical sign or a laboratory result ${ }^{11,24}$. One study evaluated global PID clinical diagnosis in patients having acute pelvic pain, but did not reliably evaluate diagnostic criteria separately ${ }^{10}$.

According to the natural history of PID, the best diagnostic test would be one with a higher sensitivity, in order to prevent sequels in real clinical practice in our emergency rooms. We need a test providing as few false negative results as possible or one with high sensitivity (to avoid the real danger of infertility resulting from late treatment). We can thus reduce the consequences of this pathology with the most complete information possible, as quickly as possible (i.e. for us - the best possible).

Adnexal tenderness has recently been described as having the best sensitivity ${ }^{25}$, whereas endocervical secretion has been previously reported as having the best specificity ${ }^{17}$. 


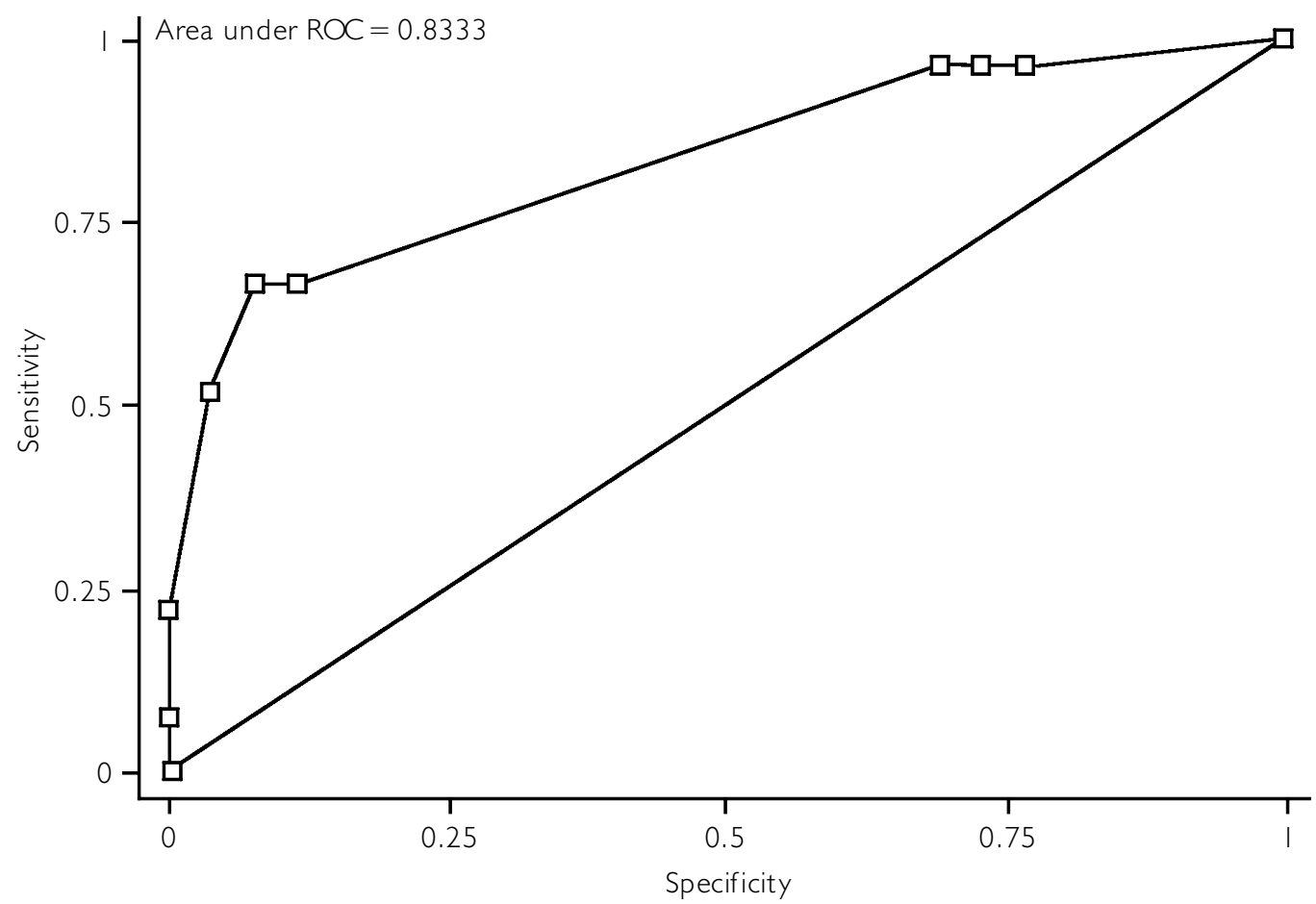

Figure 2 Receptor operative curve (ROC) for the pelvic inflammatory disease prediction model drawn up from being less than 20 years old, having purulent endocervical secretion, current use of intrauterine device and negative pregnancy test

The performance of clinical criteria in this study may have been influenced by the studied population, where lower abdominal pain symptoms were less clear. It should be recalled that sensitivity and specificity are affected by the disease's spectrum ${ }^{26}$. Concerning the operative characteristics of clinical diagnosis, Sellors ${ }^{10}$ found $52 \%$ sensitivity and $85 \%$ specificity. This difference can be explained because fallopian tube biopsy was taken as the gold standard in the aforementioned study. This may have presented a poor differential classification effect in our study, mainly affecting the group of patients with PID, as they would have been classified as being false positive, thus reducing specificity.

Even though laparoscopy is considered to be the gold standard for PID diagnosis, we saw that it gave an important number of false negatives. On comparing visual diagnosis with biopsy of the fimbria, sensitivity can fall to $65 \%{ }^{10}$. Its high specificity sustains its use as an auxiliary technique for the clinical picture in difficult cases.

Endometrial biopsy has been reported as being a key element in PID diagnosis. Its sensitivity and specificity range from 75 to $92 \%$ and 67 to $87 \%$, respectively ${ }^{12,15}$. There are differences in the gold standard used in these studies, based on visual diagnosis and microbiology of the upper genital tract.

There is no agreement respecting endometrial culture results; some authors do not agree that isolation of mycolplasma in endometrial cultures means a positive result ${ }^{27}$. Although we cannot determine causality, our results show a significant association between PID and endometrial mycoplasma-positive cultures. This finding is supported by the fact that, in some patients, mycoplasma isolation was accompanied by a diagnosis of STD. However, it might represent a marker for endometrial tissue infection.

If patients not presenting $N$. gonorroheae or anaerobic bacteria were to be excluded from analysis (ten patients in the PID group), sensitivity would rise to $50 \%(10 / 20)$ while specificity would rise to $90 \%$.

The role of transvaginal pelvic sonography in PID diagnosis is under much discussion. Its sensitivity depends on the image described, estimated at 
between 32 and 85\%, with $58-100 \%$ specificity $^{28}$, and is operator-dependent. Our results are consistent with such results.

Other prediction models have already been developed. Morcos and co-workers ${ }^{11}$, with $76 \%$ PID prevalency, found anexial pain, abdominal pain of duration less than 1 week, and high leukocyte count, to be the best predictors. Hudgu and co-workers ${ }^{10}$, with $67 \%$ PID prevalency, found that the best predictors were purulent endocervical secretion, speed of elevated globular sedimentation, and an endocervix culture for $N$. gonorrhoeae, both studies being diagnosed by laparoscopy. Peipert ${ }^{21}$, with $47 \%$ prevalency for PID diagnosed by endometrial biopsy, reported the following predictors: fever, leukocyte count greater than $10000 / \mathrm{mm}^{3}$, and a positive bacteriological test in the cervix ${ }^{21}$

Although the model's applicability is no longer useful for the whole population, it is more useful in women younger than 20 for whom the consequences of the disease can be more devastating (i.e. infertility, ectopic pregnancy, and chronic pain). Its predictive value will be most applicable to a gynecological reference service. In another scenario, prevalence ranges from $25^{29}$ to $55 \%{ }^{30}$ of those patients in a general hospital or surgical service presenting gynecological pathology as the cause of NSLAP. If we take the lower percentage as estimating lower prevalency, we see that the model would have a $67 \%$ positive predictive value and an $89 \%$ negative predictive value.

\section{CONCLUSIONS}

(1) Clinical diagnosis was the best diagnostic method for PID screening in our environment; (2) laparoscopy showed the best specificity, and is thus useful in cases having an atypical clinical course for discarding pelvic pain caused by another factor; (3) the other diagnostic methods were of limited usefulness in our study; and (4) the greater and lesser criteria evaluated in isolation presented low operative characteristics, therefore their recommended use must again be evaluated in patients having an atypical PID clinical picture.

\section{ACKNOWLEDGEMENTS}

To Beatriz Londo o and Ana Pe uela at the Bogota Health Service, for their financial and technical support; Javier Salas, Mauricio Camacho, Jaime Ruiz, Margarita Gomez, Alain Davies, Alejandro Castro and Yesid Parra in the Gynecology and Obstetrics Department of Universidad Nacional de Colombia, for their support with field work; Stella Lancheros, Carmen Garzon, Ricardo Pachon, Francisco Lopez, Consuelo Barragan, Rocio Fuquen, Luz Díaz and the Instituto Materno Infantil medical staff for their assistance in the recruitment and treatment of patients; the Hospital San Juan de Dios emergency room services medical staff, for their assistance in the recruitment and treatment of patients; and all those patients who kindly agreed to participate in our study.

\section{REFERENCES}

1. Gaitan H, Angel E, Sánchez J, et al. Laparoscopic diagnosis of acute lower abdominal pain in women of reproductive age. Int J Obstet Gynecol 2002;76: 149-58

2. Gogate A, Brabin L, Nicholas S, et al. Risk factors for laparoscopically confirmed pelvic inflammatory disease: findings from Mumbai (Bombay), India. Sex Transm Infect 1998;74:426-32

3. Gaitán H, Angel E, Medina M, et al. Diagnóstico de la enfermedad pélvica inflamatoria en el Instituto Materno Infantil 1992-93. Revista Facultad de Medicina, Universidad Nacional 1996;44:134-41
4. Quazi MA. Lethal outcome of pelvic inflammatory disease in five women who were seropositive for HIV. Eur J Surg 1996;162:67-9

5. Buchan H, Vessey M, Goldacre M, Faireweather J. Morbidity following pelvic inflammatory disease. Br J Obstet Gynecol 1993;100:558-62

6. Lepine LA, Hillis SD, Marchbanks PA, et al. Severity of pelvic inflammatory disease as a predictor of the probability of live birth. Am J Obstet Gynecol 1998;178:977-81 
7. Jacobson L, Weström L. Objectivized diagnosis of acute pelvic inflammatory disease. Am J Obstet Gynecol 1969;105:1088-98

8. McCormack WM. Pelvic inflammatory disease. New Eng J Med 1994;330:115-19

9. Sellors J, Mahony J, Goldsmith C, et al. The accuracy of clinical findings and laparoscopy in pelvic inflammatory disease. Am J Obstet Gynecol 1991; 164:113-20

10. CDC. Pelvic Inflammatory Disease: guidelines for prevention and management. Morbid Mortal Wkly Rep 1991;40:1-25

11. Hudgu A, Westrom L, Brooks C, et al. Predicting acute pelvic inflammatory disease. A multivariate analysis. Am J Obstet Gynecol 1986;155:954-60

12. Morocos R, Frost N, Hnat M, et al. Laparoscopy versus clinical diagnosis of acute pelvic inflammatory disease. J Reproductive Med 1993;38:53-6

13. Paavonen J, Aine R, Teisala K, et al. Comparison of endometrial biopsy and peritoneal fluid with laparoscopy in the diagnosis of acute pelvic inflammatory disease. Am J Obstet Gynecol 1985;151: $645-50$

14. Weiss N. Clinical epidemiology. In: Rothman K, Greendland S. Modern Epidemiology 2nd edn. Philadelphia: Lippincott, Williams and Wilkins, 1998

15. Hager WD, Eschembach DA, Spence MR, Sweet RL. Criteria for diagnosis and grading of salpingitis. Obstet Gynecol 1983;61:113-14

16. Llanio R. Laparoscopia de urgencias. 1a edición. La Habana: Editorial científico técnica, 1977

17. Soper D. Diagnosis and laparoscopic grading of acute salpingitis. Am J Obstet Gynecol 1991;164: $1370-6$

18. Kiviat N, Wolner-Hanssen P, Eschembach D, et al. Endometrial histopathology in patients with culture-proved upper genital tract infection and laparoscopically diagnosed acute salpingitis. $A m \mathrm{~J}$ Surg Pathol 1990;14:167-75

19. Arredondo JL, Diaz V, Gaitan H, et al. Oral Clindamycin and Ciprofloxacin versus intramuscular Ceftriaxone and oral Doxycycline in the treatment of mild to moderate pelvic inflammatory disease in outpatients. Clin Infet Dis 1997;24:170-8

20. Echeverry J, Ardila E. Pruebas diagnosticas y proceso diagnostico. In: Ardila E, Sánchez R, Echeverry J. Estrategias de Investigación en Medicina Clínica. Bogotá: Manual Moderno, 2001

21. Boardman L, Peipert JF, Brody J, et al. Endovaginal sonography for the diagnosis of upper genital tract infection. Obstet Gynecol 1997;90:54-7

22. Garret J. Quantitative Methods Logistic Regression, Survival Analysis and Poisson Regression. 1995.

23. Roper W, Winkenwerder W, Hackbarth G, Krakauer H. Effectiveness in health care. An initiative to evaluate and improve medical practice. New Engl J Med 1988:319:1197-202

24. Livengood C 3rd, Hill G, Addison W. Pelvic inflammatory disease: findings during in-patient treatment of clinically severe, laparoscopydocumented disease. Am J Obstet Gyencol 1992; 166:519-24

25. Peipert J, Ness R, Blume J, et al. Clinical predictors of endometritis in women with symptoms and signs of pelvic inflammatory disease. Am J Obstet Gynecol 2001;184:856-63

26. Rfansohoff D, Feinstein A. Problems of spectrum and bias in evaluating the efficacy of diagnostic tests. N Engl J Med 1978;299:926-30

27. Hillier SL, Kiviat NB, Hawes SE, et al. Role of bacterial vaginosis-associated microorganisms in endometritis. Am J Obstet Gynecol 1996;175: $435-41$

28. Cicatore B, Leminen A, Ingman-Friberg S, et al. Transvaginal sonographic findings in ambulatory patients with suspected pelvic inflammatory disease. Obstet Gynecol 1992;80:912-16

29. Decadt B, Sussman L, Lewis M, et al. Randomised clinical trial of early laparoscopy in the management of acute non-specific abdominal pain. $\mathrm{Br} J$ Surg 1999;86:1383-6

30. Sugarbaker P, Bloom B, Sanders J, Wilson R. Preoperative laparoscopy in diagnosis of acute pelvic pain. Lancet 1975;442-5

RECEIVED 03/04/02; ACCEPTED 09/03/02 


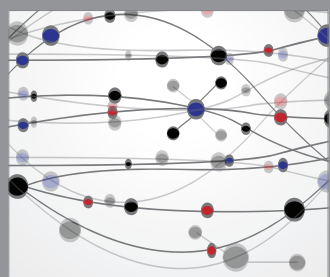

The Scientific World Journal
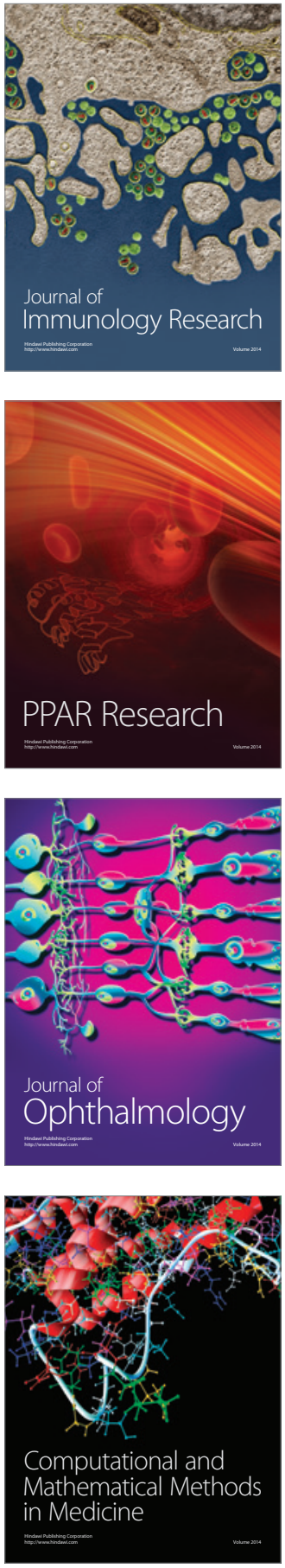

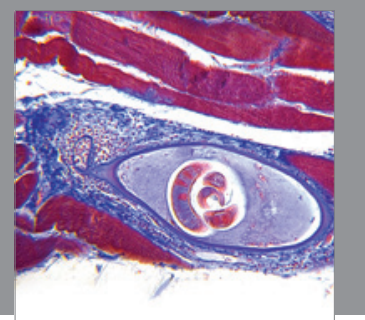

Gastroenterology

Research and Practice
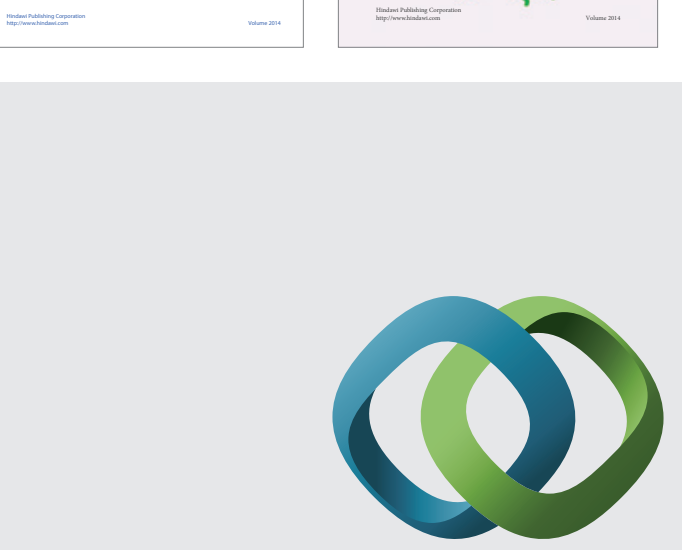

\section{Hindawi}

Submit your manuscripts at

http://www.hindawi.com
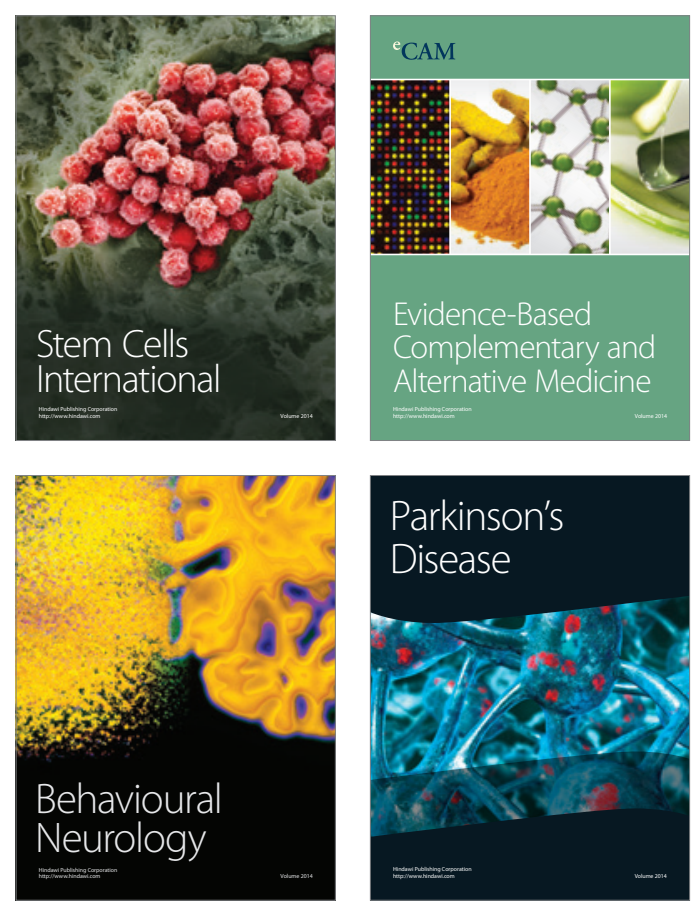

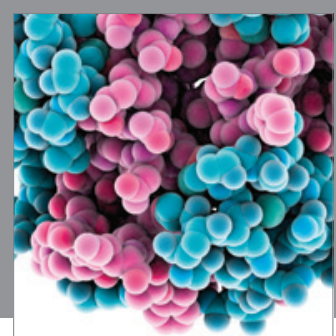

Journal of
Diabetes Research

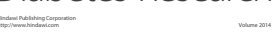

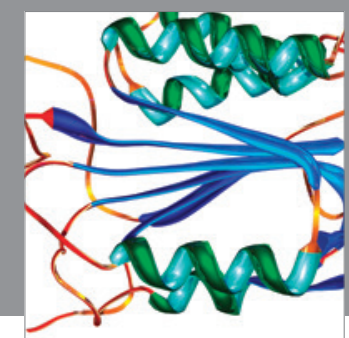

Disease Markers
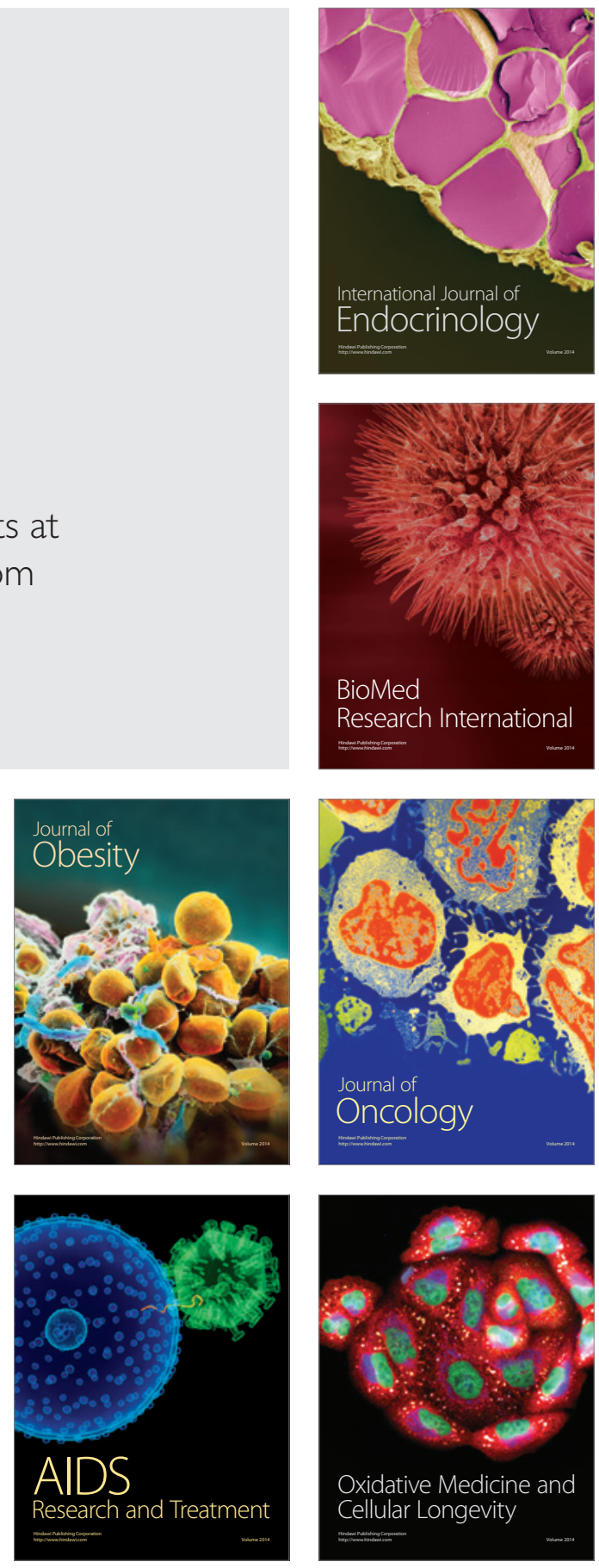